\title{
On the real roots of generalized Thue-Morse polynomials
}

\author{
by \\ Christophe Doche (Talence)
}

In this article we investigate real roots of real polynomials. By results of M. Kac [8-10] we know that a polynomial of degree $n$ has on average $(2 / \pi) \log n$ real zeros. See also results of Edelman and Kostlan [6] on the same subject. Some 10 years later Erdös and Offord [7] proved that the mean number of real roots of a random polynomial of degree $n$ with coefficients \pm 1 is again $(2 / \pi) \log n$. This leads us to the following question: can we find sequences $\left(\alpha_{i}\right)_{i \in \mathbb{N}}$ with coefficients \pm 1 such that the corresponding polynomials $\sum_{i=0}^{n} \alpha_{i} X^{i}$ have $O(\log n)$ real roots, and are these sequences random in some sense?

We introduce generalized Thue-Morse sequences whose corresponding polynomials of large degree $n$ have at least $C \log n$ real roots, where $C$ is an explicit positive constant. Finally, we discuss the spectral measure of these sequences.

1. Introduction. Erdös and Offord [7] established that the average number of real zeros of the degree $n$ polynomial $\sum_{i=0}^{n} \pm X^{i}$ is equivalent to $(2 / \pi) \log n$. A natural question could then be to find a sequence $\left(\alpha_{i}\right)_{i \in \mathbb{N}}$ of \pm 1 such that

$$
\frac{1}{N} \sum_{n=0}^{N-1} \varrho\left(g_{n}\right) \backsim \frac{2}{\pi} \log N
$$

where $\varrho\left(g_{n}\right)$ is the number of real zeros of the polynomial

$$
g_{n}(X)=\sum_{i=0}^{n} \alpha_{i} X^{i} .
$$

In a previous article [5] we tested the Thue-Morse sequence $\left(\varepsilon_{i}\right)_{i \in \mathbb{N}}$, defined by $\varepsilon_{i}=(-1)^{\nu(i)}$ where $\nu(i)$ is the sum of the binary digits of $i$.

2000 Mathematics Subject Classification: Primary 12D10.

Key words and phrases: real roots of polynomials, Thue-Morse sequence, spectral measure. 
Unfortunately, we proved that (1) does not hold in this case. More precisely we have

$$
\frac{1}{N} \sum_{n=0}^{N-1} \varrho\left(f_{n}\right) \underset{N \rightarrow \infty}{\longrightarrow} \frac{11}{4}
$$

with $f_{n}(X)=\sum_{i=0}^{n} \varepsilon_{i} X^{i}$.

In this paper we show the existence of families $\left(\varepsilon_{w, i}\right)_{i \in \mathbb{N}}$ of $( \pm 1)$-sequences for which

$$
\liminf _{N \rightarrow \infty} \frac{1}{N \log N} \sum_{n=0}^{N-1} \varrho\left(f_{w, n}\right)>0,
$$

where $f_{w, n}(X)=\sum_{i=0}^{n} \varepsilon_{w, i} X^{i}$. These sequences have, to some extent, a similar structure to the Thue-Morse sequence. They can be obtained in a very similar way, by means of iterations of morphisms. We call them generalized Thue-Morse sequences.

Before explaining this we introduce two useful notations. Let $w$ be a word of length $\ell$ on the alphabet $\{+,-\}$ and $i \leq j$ be integers less than $\ell$. Then ${ }_{i} w_{j}$ represents the factor of $w$ beginning at letter $i$ and finishing at letter $j$ of $w$. For instance ${ }_{i} w_{i}$ is simply the letter at position $i$ of $w$ and ${ }_{0} w_{\ell-1}=w$. We put also $w[i]=1$ if ${ }_{i} w_{i}=+$ and $w[i]=-1$ if ${ }_{i} w_{i}=-$.

Now let $\varphi$ be the morphism on the alphabet $\{+,-\}$ defined by

$$
\varphi:\left\{\begin{array}{l}
+\rightarrow+- \\
-\rightarrow-+
\end{array}\right.
$$

The first iterations of $\varphi$ are

$$
\begin{aligned}
\varphi(+) & =+- \\
\varphi^{2}(+) & =+--+, \\
\varphi^{3}(+) & =+--+-++-.
\end{aligned}
$$

Let

$$
\mathcal{E}=\lim _{n \rightarrow \infty} \varphi^{n}(+)
$$

be the Thue-Morse word. There exists an obvious link between $\mathcal{E}$ and $\left(\varepsilon_{i}\right)_{i \in \mathbb{N}}$, i.e.

$$
\varepsilon_{i}=\mathcal{E}[i]
$$

In the next section we slightly modify the definition of $\varphi$ to get a wider family of sequences.

2. Generalized Thue-Morse sequences. Let $w$ be a word on $\{+,-\}$ of length $\ell \geq 2$ beginning with + . We put

$$
\varphi_{w}:\left\{\begin{array}{l}
+\rightarrow w \\
-\rightarrow \bar{w}
\end{array}\right.
$$


where $\bar{w}$, the "opposite" of $w$, is defined by

$$
{ }_{i} \bar{w}_{i}= \begin{cases}- & \text { if }{ }_{i} w_{i}=+, \\ + & \text { if }{ }_{i} w_{i}=-\end{cases}
$$

The Thue-Morse $w$-word, $\mathcal{E}_{w}$, is then

$$
\mathcal{E}_{w}=\lim _{n \rightarrow \infty} \varphi_{w}^{n}(+) .
$$

Denote by $\left(\varepsilon_{w, i}\right)_{i \in \mathbb{N}}$ the corresponding Thue-Morse $w$-sequence with coefficients \pm 1 , that is, the sequence satisfying

$$
\varepsilon_{w, i}=\mathcal{E}_{w}[i] .
$$

For example if $w=++$ then

$$
\sum_{i=0}^{\infty} \varepsilon_{w, i} X^{i}=\frac{1}{1-X} .
$$

For each word $w$ of length $\ell$ we consider $P_{w}$ its associated polynomial defined by

$$
P_{w}(X)=\sum_{j=0}^{\ell-1} w[j] X^{j} .
$$

LEMMA 1. Let $w$ be a word of length $\ell \geq 2$ on $\{+,-\}$ beginning with + and $\varepsilon_{w}$ its associated generalized Thue-Morse sequence. Then

$$
\sum_{i=0}^{\infty} \varepsilon_{w, i} X^{i}=\prod_{h=0}^{\infty} P_{w}\left(X^{\ell^{h}}\right) .
$$

Proof. Let $v$ be some word of length $t$ on $\{+,-\}$ and

$$
P_{v}(X)=\sum_{j=0}^{t-1} v[j] X^{j}
$$

its associated polynomial. It suffices to see that

$$
P_{\varphi_{w}(v)}(X)=P_{w}(X) P_{v}\left(X^{\ell}\right)
$$

Indeed,

$$
{ }_{j \ell}\left(\varphi_{w}(v)\right)_{j \ell+\ell-1}= \begin{cases}w & \text { if } v[j]=1, \\ \bar{w} & \text { if } v[j]=-1,\end{cases}
$$

for all $j$ in $\llbracket 0, t-1 \rrbracket$. So we get

$$
P_{\varphi_{w}(v)}(X)=\sum_{j=0}^{t-1} P_{w}(X) v[j] X^{j \ell}
$$

and $P_{\varphi_{w}(v)}(X)=P_{w}(X) P_{v}\left(X^{\ell}\right)$ as claimed. The lemma immediately follows. Note that the series converges in $]-1,1[$. 
Using this we can give for $\varepsilon_{w, i}$ a more explicit meaning that generalizes the initial definition of the Thue-Morse sequence. Let $m_{1}, \ldots, m_{q}$ be the integers $j \in \llbracket 0, \ell-1 \rrbracket$ such that ${ }_{j} w_{j}=-$. Then from (2) it is clear that

$$
\varepsilon_{w, i}=(-1)^{\nu_{m_{1}}(i)+\ldots+\nu_{m_{q}}(i)}
$$

where $\nu_{m_{k}}(i)$ represents the number of $m_{k}$ 's in the base $\ell$ expansion of $i$.

3. Real roots of generalized Thue-Morse polynomials. For the classical Thue-Morse polynomials the starting word is $w=+-$ so that its associated polynomial is $P_{w}(X)=1-X$. Therefore $\ell=2$ and since the real roots of $P_{w}\left(X^{2^{h}}\right)$ are -1 and 1 , we cannot use the convergence of $\sum_{i=0}^{\infty} \varepsilon_{i} X^{i}$ on $]-1,1\left[\right.$. Now the starting polynomial $P_{w}$ may vanish on $] 0,1[$ and the real roots of $P_{w}\left(X^{\ell^{h}}\right)$ spread in this case along [0,1[. Using the convergence of (2) on $]-1,1\left[\right.$ we show that $\varrho\left(f_{w, n}\right)$ is in $C \log n$. Let us make this more precise.

Theorem 1. Let $w$ be a word of length $\ell \geq 2$ on $\{+,-\}$ beginning with + such that $P_{w}$ has only simple roots on $]-1,1\left[\right.$, say $t$ roots $\beta_{1}<\ldots<\beta_{t}$ in $] 0,1\left[\right.$ and $t^{\prime}$ roots $\beta_{t+t^{\prime}}<\ldots<\beta_{t+2}<\beta_{t+1}$ in $]-1,0\left[\right.$. Let $f_{w, n}$ be the generalized Thue-Morse polynomials associated with $\varepsilon_{w}$. Assume that $\beta_{t}<\beta_{1}^{1 / \ell}$. Suppose in addition that $\beta_{t+1}^{1 / \ell}<\beta_{t+t^{\prime}}$ if $\ell$ is odd. Then there exists $K>0$ such that for all $\epsilon>0$ there is an $N(\epsilon)$ such that for all $n \geq N(\epsilon)$ we have

$$
\begin{array}{ll}
\varrho\left(f_{w, n}\right) \geq \frac{2(1-\epsilon) t \log n}{\log \ell}-K & \text { if } \ell \text { is even, } \\
\varrho\left(f_{w, n}\right) \geq \frac{(1-\epsilon)\left(t+t^{\prime}\right) \log n}{\log \ell}-K & \text { if } \ell \text { is odd. }
\end{array}
$$

Proof. Put $\beta_{j, h}=\beta_{j}^{1 / \ell^{h}}$ for all $h \in \mathbb{Z}$. The roots of

$$
f_{w, \ell^{k}-1}(X)=\prod_{h=0}^{k-1} P_{w}\left(X^{\ell^{h}}\right)
$$

in $] 0,1[$ are therefore

$$
\begin{array}{ccc}
\beta_{1,0} & \ldots & \beta_{t, 0}, \\
\vdots & & \vdots \\
\beta_{1, h} & \ldots & \beta_{t, h}, \\
\vdots & & \vdots \\
\beta_{1, k-1} & \ldots & \beta_{t, k-1} .
\end{array}
$$

Put also

$$
\delta_{j, h}= \begin{cases}\sqrt{\beta_{j, h} \beta_{j+1, h}} & \text { for } j \in \llbracket 1, t-1 \rrbracket, h \in \mathbb{Z}, \\ \sqrt{\beta_{t, h} \beta_{1, h+1}} & \text { for } j=t, h \in \mathbb{Z} .\end{cases}
$$


The next lemma plays an important part in the following.

Lemma 2. Let $u$ be the multiplicity of 1 as a root of $P_{w}$. Then there are two constants $C_{1}$ and $C_{2}$ such that for all large $h, k \geq h$ and $j \in \llbracket 1, t \rrbracket$,

$$
\left|f_{w, \ell^{k}-1}\left(\delta_{j, h}\right)\right| \geq C_{1} C_{2}^{h} \ell^{-u h(h+1) / 2} .
$$

Proof of Lemma 2. First of all we determine $C_{1}(j)$ and $C_{2}(j)$ for any $j$ in $\llbracket 1, t \rrbracket$. Since there are only finitely many $j$ 's the lemma will follow immediately.

We remark that

$$
f_{w, \ell^{k}-1}\left(\delta_{j, h}\right)=\prod_{s=0}^{k-1} P_{w}\left(\delta_{j, h}^{\ell^{s}}\right)=\prod_{i=h-k+1}^{h} P_{w}\left(\delta_{j, i}\right) .
$$

Now

$$
P_{w}\left(\delta_{j, i}\right)=\frac{P_{w}^{(u)}(1)}{u !}\left(1-\delta_{j, i}\right)^{u}+o\left(\left(1-\delta_{j, i}\right)^{u}\right)
$$

when $1-\delta_{j, i} \rightarrow 0$, so there are $C_{5} \in \mathbb{R}_{+}$and $i_{0}>0$ such that for all $i>i_{0}$,

$$
\left|P_{w}\left(\delta_{j, i}\right)\right| \geq C_{5}\left|1-\delta_{j, i}\right|^{u} \geq C_{5}\left|\frac{\log \delta_{j, 0}}{2 \ell^{i}}\right|^{u}
$$

since $\left|1-e^{x}\right| \geq|x| / 2$ near 0 . Thus

$$
\prod_{i=i_{0}}^{h}\left|P_{w}\left(\delta_{j, i}\right)\right| \geq C_{4} C_{3}^{h} \ell^{-u h(h+1) / 2} .
$$

The factor

$$
\prod_{i=h-k+1}^{i_{0}-1}\left|P_{w}\left(\delta_{j, i}\right)\right|
$$

leads us to study the behaviour of $P_{w}$ near 0 . Now $P_{w}$ is locally either greater than 1 or less than 1 . In the first case it is obvious that for a suitable constant $C_{1}$,

$$
\prod_{i=h-k+1}^{h}\left|P_{w}\left(\delta_{j, i}\right)\right| \geq C_{1} C_{2}^{h} \ell^{-u h(h+1) / 2} .
$$

In the second case $\delta_{j, i} \geq \delta_{j, i}^{2} \geq \ldots \geq \delta_{j, i}^{\ell-1}$ and these quantities are small in comparison with 1 for all large $|i|$. Thus

$$
\left|P_{w}\left(\delta_{j, i}\right)-1\right| \leq(\ell-1) \delta_{j, i}
$$

Since for $i \leq 0$ we have $\delta_{j, i}=\delta_{j, 0}^{\ell^{|i|}}$, we obtain the convergence of

$$
\prod_{i=-\infty}^{i_{0}-1}\left|P_{w}\left(\delta_{j, i}\right)\right|
$$

The lemma is then proved. 
Remark. When $P_{w}(1) \neq 0$ it is possible to replace $C_{1} C_{2}^{h} \ell^{-u h(h+1) / 2}$ in (3) by a positive constant independent of $h$.

Since $f_{w, \ell^{k}-1}$ has $t-1$ simple roots between $\delta_{1, h}$ and $\delta_{t, h}$, it follows that $f_{w, \ell^{k}-1}$ changes sign $t-1$ times, passing above and below the lines

$$
y=C_{1} C_{2}^{h} \ell^{-u h(h+1) / 2} \text { and } y=-C_{1} C_{2}^{h} \ell^{-u h(h+1) / 2} .
$$

For example, consider Figure 1 which displays $f_{w, 728}$ built from the word $w=+--$. As $P_{w}(1)=-1$ the remark ensures that $f_{w, 728}$ and more generally $f_{w, 3^{k}-1}$ winds itself round two absolute axes. Here they are $y=$ 0.067130 and $y=-0.067130$ (bold lines on Figure 1).

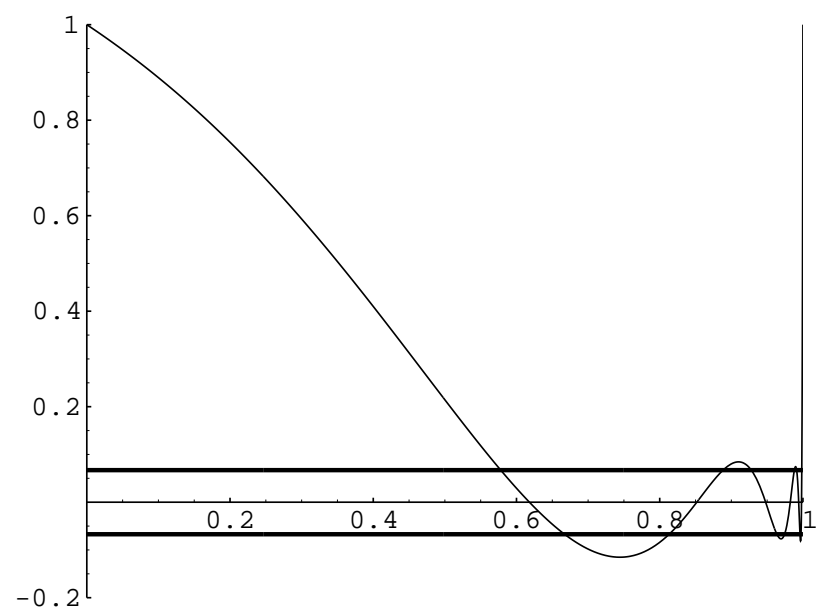

Fig. 1. $f_{w, 728}(X)$ on $[0,1]$

Now if $n \geq \ell^{k}$ it is clear that on $] 0,1[$,

$$
\left|f_{w, n}(x)-f_{w, \ell^{k}-1}(x)\right| \leq \frac{x^{\ell^{k}}}{1-x} .
$$

Let $\epsilon>0$. Since $\delta_{t,\lfloor(1-\epsilon) k\rfloor}^{\ell^{k}}=\delta_{t,\lfloor(1-\epsilon) k\rfloor-k} \leq \delta_{t}^{\ell^{\epsilon k}}$, we have

$$
\frac{\delta_{t,\lfloor(1-\epsilon) k\rfloor}^{\ell^{k}}}{1-\delta_{t,\lfloor(1-\epsilon) k\rfloor}} \cdot \frac{\ell^{u k(k+1) / 2}}{C_{1} C_{2}^{k}} \underset{k \rightarrow \infty}{\longrightarrow} 0 .
$$

So there is $k(\epsilon)$ such that for all $k \geq k(\epsilon)$,

$$
\delta_{t,\lfloor(1-\epsilon) k\rfloor}^{\ell^{k}}-C_{1} C_{2}^{k} \ell^{-u k(k+1) / 2}\left(1-\delta_{t,\lfloor(1-\epsilon) k\rfloor}\right) \leq 0 .
$$

Moreover the function $x^{\ell^{k}}-C_{1} C_{2}^{k} \ell^{-u k(k+1) / 2}(1-x)$ is increasing on $] 0,1[$. 
So on $\left.] 0, \delta_{t,\lfloor(1-\epsilon) k\rfloor}\right]$

$$
\frac{x^{\ell^{k}}}{1-x}<C_{1} C_{2}^{k} \ell^{-u k(k+1) / 2} .
$$

This inequality ensures that $f_{w, n}$ is subject to the same oscillations as $f_{w, \ell^{k}-1}$ provided Lemma 2 holds. Indeed, for $h$ and $k$ large,

$$
f_{w, n}\left(\delta_{j, h}\right) \geq f_{w, \ell^{k}-1}\left(\delta_{j, h}\right)-\frac{x^{\ell^{k}}}{1-x}>0
$$

when $f_{w, \ell^{k}-1}\left(\delta_{j, h}\right)>0$ and

$$
f_{w, n}\left(\delta_{j, h}\right) \leq f_{w, \ell^{k}-1}\left(\delta_{j, h}\right)+\frac{x^{\ell^{k}}}{1-x}<0
$$

when $f_{w, \ell^{k}-1}\left(\delta_{j, h}\right)<0$.

Let $\varrho_{1}\left(f_{w, n}\right)$ be the number of real roots of $f_{w, n}$ in $] 0,1[$. So

$$
\varrho_{1}\left(f_{w, n}\right) \geq \frac{(1-\epsilon) t \log n}{\log \ell}-K_{1},
$$

for a suitable absolute constant $K_{1}$, as soon as $n \geq N(\epsilon)=\ell^{k(\epsilon)}$.

If $\ell$ is even then $f_{w, n}$ has at least $\lfloor(1-\epsilon) k\rfloor t$ roots in ]-1,0[ for large $k$ and $n \geq \ell^{k}$. Then

$$
\varrho\left(f_{w, n}\right) \geq \frac{2(1-\epsilon) t \log n}{\log \ell}-K
$$

for all large $n$.

If $\ell$ is odd we apply what we have just seen to $P_{w}(-X)$. This polynomial has by hypothesis $t^{\prime}$ roots in $] 0,1\left[\right.$ so that $f_{w, n}(-X)$ has at least $\lfloor(1-\epsilon) k\rfloor t^{\prime}$ roots in $] 0,1[$. Thus

$$
\varrho\left(f_{w, n}\right) \geq \frac{(1-\epsilon)\left(t+t^{\prime}\right) \log n}{\log \ell}-K,
$$

for all large $n$.

This ends the proof of Theorem 1.

Under the assumptions of Theorem 1 we have the following inequalities.

Corollary. If $\ell$ is even then

$$
\liminf _{N \rightarrow \infty} \frac{1}{N \log N} \sum_{n=0}^{N-1} \varrho\left(f_{w, n}\right) \geq \frac{2 t}{\log \ell} .
$$

If $\ell$ is odd then

$$
\liminf _{N \rightarrow \infty} \frac{1}{N \log N} \sum_{n=0}^{N-1} \varrho\left(f_{w, n}\right) \geq \frac{t+t^{\prime}}{\log \ell} .
$$


Proof. Assuming $\ell$ to be even, we apply Theorem 1 to obtain

$$
\begin{aligned}
\frac{1}{N \log N} \sum_{n=0}^{N-1} \varrho\left(f_{w, n}\right) & \geq \frac{1}{N \log N} \cdot \frac{2(1-\epsilon) t}{\log \ell}\left(\sum_{n=N(\epsilon)}^{N-1} \log n-N K\right) \\
& \geq \frac{2(1-\epsilon) t}{\log \ell}-\frac{K^{\prime}}{\log N} .
\end{aligned}
$$

When $N$ tends to infinity we get

$$
\liminf _{N \rightarrow \infty} \frac{1}{N \log N} \sum_{n=0}^{N-1} \varrho\left(f_{w, n}\right) \geq \frac{2(1-\epsilon) t}{\log \ell} .
$$

Since this is true for all $\epsilon>0$ we deduce that

$$
\liminf _{N \rightarrow \infty} \frac{1}{N \log N} \sum_{n=0}^{N-1} \varrho\left(f_{w, n}\right) \geq \frac{2 t}{\log \ell} .
$$

If $\ell$ is odd the same argument works.

So generalized Thue-Morse sequences yield polynomials with many real roots; but what can we say on their random behaviour? In the next section we say a few words about this.

\section{Spectral measure of the generalized Thue-Morse sequences.}

A good tool to evaluate the random nature of a sequence is to study its spectral measure defined for instance in [11] and in [1]. We recall basic definitions, and then we give just the important results without all intermediate steps.

Let $\gamma_{w}(h)$ be the correlation function of $\left(\varepsilon_{w, n}\right)_{n \in \mathbb{N}}$ defined by

$$
\gamma_{w}(h)=\lim _{N \rightarrow \infty} \frac{1}{N} \sum_{n=0}^{N-1} \varepsilon_{w, n} \varepsilon_{w, n+h} .
$$

This limit always exists for generalized Thue-Morse sequences. The spectral measure $d \sigma_{w}$ is linked to $\gamma_{w}(h)$ by the formula

$$
\gamma_{w}(h)=\int_{0}^{1} e^{2 i \pi h x} d \sigma_{w}(x) .
$$

For all $i \in \llbracket 1, \ell-1 \rrbracket, \gamma_{w}$ satisfies the recurrence relations

$$
\begin{aligned}
\gamma_{w}(\ell k) & =\gamma_{w}(k), \\
\gamma_{w}(\ell k+i) & =\frac{a_{w, i}}{\ell} \gamma_{w}(k)+\frac{a_{w, \ell-i}}{\ell} \gamma_{w}(k+1),
\end{aligned}
$$

where $a_{w, i}=\sum_{j=0}^{\ell-i-1} w[j] w[i+j]$. 
To establish that $d \sigma_{w}$ is continuous we know [1] that it is sufficient to prove that

$$
\lim _{N \rightarrow \infty} \frac{1}{N} \sum_{n=0}^{N-1} \gamma_{w}(n)^{2}=0
$$

Let

$$
\Gamma_{w}(h)=\lim _{N \rightarrow \infty} \frac{1}{N} \sum_{m<N} \gamma_{w}(m) \gamma_{w}(m+h),
$$

and we are left to show $\Gamma_{w}(0)=0$. Following the ideas of $[1$, Appendix I] we obtain the system

$$
\mathcal{S}:\left\{\begin{array}{l}
c_{11}(w) \Gamma_{w}(0)+c_{12}(w) \Gamma_{w}(1)=0 \\
c_{21}(w) \Gamma_{w}(0)+c_{22}(w) \Gamma_{w}(1)=0
\end{array}\right.
$$

where

$$
\begin{aligned}
& c_{11}(w)=1-\frac{1}{\ell}-2 \sum_{j=1}^{\ell-1} \frac{a_{w, j}^{2}}{\ell^{3}}, \\
& c_{12}(w)=-2 \sum_{j=1}^{\ell-1} \frac{a_{w, j} a_{w, \ell-j}}{\ell^{3}}, \\
& c_{21}(w)=\frac{2 a_{w, 1}}{\ell^{2}}+2 \sum_{j=1}^{\ell-2} \frac{a_{w, j} a_{w, j+1}}{\ell^{3}}, \\
& c_{22}(w)=-1+\frac{2 a_{w, \ell-1}}{\ell^{2}}+\sum_{j=1}^{\ell-2} \frac{a_{w, j+1} a_{w, \ell-j}+a_{w, j} a_{w, \ell-j-1}}{\ell^{3}} .
\end{aligned}
$$

LEMMA 3. Let $w$ be word of length $\ell \geq 2$. We say $w$ is of type ++ if $w=+\ldots+(\ell$ times $)$, and of type +-+ if $\ell$ is odd and $w=+(-+) \ldots(-+)$ $((\ell-1) / 2$ brackets $)$. Then the determinant $\Delta(w)$ of $\mathcal{S}$ vanishes if and only if $w$ is of type ++ or +-+ .

Proof. It is almost immediate that

$$
\begin{array}{rlrl}
\left|c_{11}(w)\right| & \geq \frac{\ell^{3}-\ell}{3 \ell^{3}}, & \left|c_{12}(w)\right| \leq \frac{\ell^{3}-\ell}{3 \ell^{3}} \\
\left|c_{21}(w)\right| \leq \frac{2 \ell^{3}-2 \ell}{3 \ell^{3}}, & \left|c_{22}(w)\right| \geq \frac{2 \ell^{3}-2 \ell}{3 \ell^{3}} .
\end{array}
$$

For example, let us show the last inequality. We know that

$$
c_{22}(w)=-1+\frac{2 a_{w, \ell-1}}{\ell^{2}}+\sum_{j=1}^{\ell-2} \frac{a_{w, j+1} a_{w, \ell-j}+a_{w, j} a_{w, \ell-j-1}}{\ell^{3}} .
$$


Since

$$
\begin{aligned}
\left|\sum_{j=1}^{\ell-2} \frac{a_{w, j+1} a_{w, \ell-j}+a_{w, j} a_{w, \ell-j-1}}{\ell^{3}}\right| & \leq \sum_{j=1}^{\ell-2} \frac{(\ell-j-1) j+(\ell-j)(j+1)}{\ell^{3}} \\
& \leq \frac{\ell^{3}-4 \ell}{3 \ell^{3}}
\end{aligned}
$$

we have

$$
\left|c_{22}(w)\right| \geq 1-\frac{2\left|a_{w, \ell-1}\right|}{\ell^{2}}-\frac{\ell^{3}-4 \ell}{3 \ell^{3}} \geq \frac{2 \ell^{3}-2 \ell}{3 \ell^{3}} .
$$

We also notice that the last inequality is an equality if and only if

$$
\left\{\begin{array}{l}
\left|a_{w, j}\right|=\ell-j \quad \text { for } j \in \llbracket 0, \ell-1 \rrbracket, \\
a_{w, \ell-1}=1 .
\end{array}\right.
$$

If (4) is not satisfied, then $\left|c_{22}(w)\right|>\left(2 \ell^{3}-2 \ell\right) /\left(3 \ell^{3}\right)$ and

$$
|\Delta(w)| \geq\left|c_{11}(w) c_{22}(w)\right|-\left|c_{12}(w) c_{21}(w)\right|>0,
$$

which implies that $\Gamma_{w}(0)=0$. Now if (4) holds then $w[j] w[j+1]$ does not depend on $j \in \llbracket 0, \ell-2 \rrbracket$. Therefore $w[j]=1$ or $w[j]=(-1)^{j}$ for all $j \in \llbracket 0, \ell-1 \rrbracket$. When $w[j]=(-1)^{j}$ the relation $a_{w, \ell-1}=w[0] w[\ell-1]=1$ shows that $\ell$ is necessarily odd. Obviously if $w$ is of type ++ or +-+ then $\Delta(w)=0$, so that the result is proved.

This lemma ensures that the spectral measure of a generalized ThueMorse sequence is continuous, except for trivial ++ or +-+ cases. However, although continuous, $d \sigma_{w}$ is singular (see Theorem 6 of [4]). Therefore $d \sigma_{w}$ is not absolutely continuous, which would be a true random behaviour. Nonetheless this ensures that $\left(\varepsilon_{w, n}\right)_{n \in \mathbb{N}}$ is pseudo-random in the sense of Bass [2] and Bertrandias [3].

Finally, when $w$ is of type ++ its spectral measure is the Dirac mass $\delta_{0}(x)$. If $w$ is of type +-+ , then $d \sigma_{w}$ is $\delta_{1 / 2}(x)$.

Acknowledgements. I am grateful to my supervisor, L. Habsieger, for his continuous help and to M. Mendès France for his helpful comments.

\section{References}

[1] J.-P. Allouche and M. Mendès France, Automata and automatic sequences, in: Beyond Quasicrystals, F. Axel and D. Gratias (eds.), les Éditions de Physique et Springer, 1995, 293-367.

[2] J. Bass, Suites uniformément denses, moyennes trigonométriques, fonctions pseudoaléatoires, Bull. Soc. Math. France 85 (1959), 1-69.

[3] J.-P. Bertrandias, Espaces de fonctions bornées et continues en moyenne asymptotique d'ordre p, Bull. Soc. Math. France Mém. 5 (1966). 
[4] J. Coquet, T. Kamae et M. Mendès France, Sur la mesure spectrale de certaines suites arithmétiques, Bull. Soc. Math. France 105 (1977), 369-384.

[5] C. Doche and M. Mendès France, Integral geometry and real zeros of Thue-Morse polynomials, Experiment. Math. 9 (2000), 339-350.

[6] A. Edelman and E. Kostlan, How many zeros of a random polynomial are real?, Bull. Amer. Math. Soc. (N.S.) 32 (1995), 1-37.

[7] P. Erdős and A. C. Offord, On the number of real roots of a random algebraic equation, Proc. London Math. Soc. 6 (1956), 139-160.

[8] M. Kac, On the average number of real roots of a random algebraic equation, Bull. Amer. Math. Soc. 49 (1943), 314-320 and 938.

[9] - On the average number of real roots of a random algebraic equation (II), Proc. London Math. Soc. 50 (1949), 390-408.

[10] - , Probability and Related Topics in Physical Sciences, Lectures in Appl. Math., Interscience, 1959.

[11] S. Kakutani, Strictly ergodic symbolic dynamical systems, in: Proc. of the 6th Berkeley Symposium on Mathematical Statistics and Probability (Berkeley, 1970), Vol. 2, Univ. of California Press, Berkeley, 1972, 319-326.

Laboratoire d'Algorithmique Arithmétique

Université Bordeaux I

351, cours de la Libération

F-33405 Talence Cedex, France

E-mail: cdoche@math.u-bordeaux.fr

Received on 8.10.1999

and in revised form on 18.1.2001 